\title{
A Study of Consistency in Standardized Terms Related to Audiology
}

\author{
Soo Hee Oh', Junghak Lee ${ }^{1,2}$ \\ ${ }^{1}$ Audiology Institute, Hallym University of Graduate Studies, Seoul, Korea \\ ${ }^{2}$ Department of Audiology, Hallym University of Graduate Studies, Seoul, Korea
}

\begin{abstract}
The consistent use of terms enhances effective communication, knowledge transfer, and training outcomes. Although many standards in the field of acoustics and electro-acoustics have been published, there has been no terminology standard for audiology, and consistency in the terms published has not been examined. As a preliminary work, the present study investigated consistency among definitions across different standard-setting organizations. Repeatability analysis across the data was performed to obtain baseline terms, and consistency in the term definitions repeated was examined with three criteria: consistency, partial consistency, and inconsistency. A total of 3,857 terms from 253 standards published by the International Organization for Standardization, International Electronical Committee, and American National Standards Institute was reviewed, followed by repeatability and consistency analysis. Four hundred eleven terms were repeated at least two times across the data. Consistency analysis showed $43.1 \%$ consistency, $21.4 \%$ partial consistency, and $35.5 \%$ inconsistency in the 411 terms. The results indicate the current need for improvements in the consistency of standard terms. Development of an international standard in audiology terminology would have a positive impact on various outcomes including technical documentation, communication, and product and service management. The repeated term data investigated in this study can be used as preliminary information for developing standard terminology for audiology.
\end{abstract}

Key Words: Consistency, Terminology, Standardization, Consistency.

Received: March 29, 2018 / Revised: June 8, 2018 / Accepted: June 9, 2018

Correspondence: Junghak Lee, Audiology Institute, Department of Audiology, Hallym University of Graduate Studies, 427 Yeoksam-ro, Gangnam-gu, Seoul 06197, Korea

Tel: +82-2-2051-4950 / Fax: +82-2-3453-6618 / E-mail: leejh@hallym.ac.kr

\section{INTRODUCTION}

Consistent terminology as a prerequisite in various fields of study plays an important role to establish clarity by avoiding ambiguity of context and improving stakeholder communications (Schindler, 2005; Walsh, 2006). The use of consistent terms enhances effective communication, knowledge transfer, and training outcomes. On the other hand, the use of inconsistent terminology may lead to adverse outcomes (Mullen, 2010; Oh \& Lee, 2016; Schindler, 2005; Walsh, 2005). For instance, Mullen (2010) pointed out the consequences of using inconsistent terms such as communication problems with professionals and clients, difficulties with assessment, and inaccurate documentation. Recently, the influence of inconsistent terms on multilingual translation quality and efficacy has been highlighted (Across Systems $\mathrm{GmbH,} \mathrm{2015;} \mathrm{Yin} \mathrm{et} \mathrm{al.,} \mathrm{2013).}$

Two general approaches have been suggested to establish consistency in terminology: terminology framework and terminol- ogy standard. First, a framework-based approach highlights all systematic and conceptual parameters as well as synergetic interactions and develops criteria for terms. For instance, the dynamic view of a terminology framework that Walsh (2008) suggested considers the complexity of terminology in the field of communication sciences and disorders and emphasizes the active integration of five parameters involving "referent," "purpose," "users," "culture," and "context" in the framework. On the other hand, a terminology standard approach shares common normalized vocabulary and underlies clear communication with high quality; it is a widespread method for resolving inconsistency issues involving products, processes, and services (EuroTermBank Consortium, 2006).

In recent years, global markets for audiology have increased; however, a paucity of audiology terms has been standardized and uniformity in audiology terminology has not yet been achieved, with challenging issues and a lack of professional awareness remaining as obstacles (Oh \& Lee, 2016). In the meantime, the 
International Organization for Standardization Technical Committee (TC) 43 and International Electrotechnical Commission TC 1 and TC 29 have published standards for acoustics and electroacoustics covering a portion of audiology terms. The American National Standards Institute (ANSI) has also published terminology standards in the fields of acoustics (ANSI/ASA S1.1) (American National Standards Institute, 2013) and bioacoustics (ANSI/ASA S3.2) (American National Standards Institute, 2015). Nevertheless, currently, there is no terminology standard for audiology that covers products and technical processes as well as services and systems that satisfies both the general public and professionals. In addition, consistency in standardized terms has not been examined.

The purpose of the present study is to investigate the consistency of standardized terms and definitions related to audiology across different standard-setting organizations. For consistency analysis, the repeatability of standard terms across different standard term data was checked and the consistency of repeated term definitions was examined. Analysis of consistency will expand our knowledge to understand consistency issues among standard terms and the repeated term data can be used in establishing preliminary data for terminology standards for audiology.

\section{METHODS}

Figure 1 shows the process of the consistency analysis used in this study. In step 1, 3,857 terms (Table 1) published in 253 standards were reviewed. In step 2 , repeatability analysis was performed across the data. In step 3, we examined the consistency of the term definitions from step 2 and categorized the terms into three groups, as explained below.

The purpose of repeatability analysis is to establish baseline data for consistency analysis. For repeatability analysis, the number of term occurrences across the standard term data (Table 1) was counted and the terms repeated in at least two different term data were identified (step 2). In step 3, definitions of the

Standard term data:
ISO TC 43, IEC TC 29, IEC 60050-801 (2001) \& ANSI
Process
Step 1: Review a total of 3,857 terms
Step 2: Identify standard terms repeated at least two times across different
standard organizations
Step 3: Analyze consistency of the term definitions repeated

Figure 1. Process of consistency and repeatability analysis. ISO: International Organization for Standardization, TC: technical committee, IEC: International Electrotechnical Commission, ANSI: American National Standards Institutes. repeated terms were reviewed for consistency analysis with our own criteria developed for this study. Three consistency criteria were used: complete consistency, partial consistency, and inconsistency. Complete consistency indicates 100\% identical definitions among the repeated terms and partial consistency indicates partially identical (less than 100\% identical ) definitions for the same meanings across the repeated terms. Inconsistency indicates nonidentical definitions (totally different expressions) with the same or different meanings.

\section{RESULTS}

Four hundred eleven terms repeated at least two times were identified from the data for this study. Table 2 shows the number of occurrences of the repeated terms. For instance, 340 out of 411 repeated terms occurred twice across the standard term data. Consistency analysis based on the three criteria showed $43.1 \%$ consistency, $21.4 \%$ partial consistency, and 35.5\% inconsistency in the 411 terms, indicating inconsistency issues in the standard terminology (Figure 2).

\section{DISCUSSIONS}

Standardized terminology ensures non-ambiguity, transparency, and consistency of contexts. In particular, terminology stan-

Table 1. Standard term data for consistency and repeatability analysis of this study

\begin{tabular}{cccc}
\hline $\begin{array}{c}\text { Standard } \\
\text { organizations }\end{array}$ & Standard term data & $\begin{array}{c}\text { No. of } \\
\text { standards }\end{array}$ & $\begin{array}{c}\text { No. of } \\
\text { terms }\end{array}$ \\
\hline ISO & $\begin{array}{c}\text { TC } 43 \text { acoustics published by } \\
2015\end{array}$ & 200 & 1,995 \\
IEC $\quad \begin{array}{c}\text { TC } 29 \text { electroacoustics } \\
\text { published by 2015, }\end{array}$ & 51 & 890 \\
& TC 1 60050-801 & \\
ANSI & $\begin{array}{c}\text { S1.1 terminology acoustics, } \\
\text { S3.2 terminology bioacoustics }\end{array}$ & 2 & 972 \\
\hline
\end{tabular}

ISO: International Organization for Standardization, IEC: International Electrotechnical Commission, ANSI: American National Standards Institutes, TC: technical committee

Table 2. A summary of the number of occurrences and terms repeated

\begin{tabular}{cc}
\hline Number of occurrences & Number of terms repeated \\
\hline 2 & 340 \\
3 & 49 \\
4 & 17 \\
5 & 5 \\
Total terms repeated & 411 \\
\hline
\end{tabular}




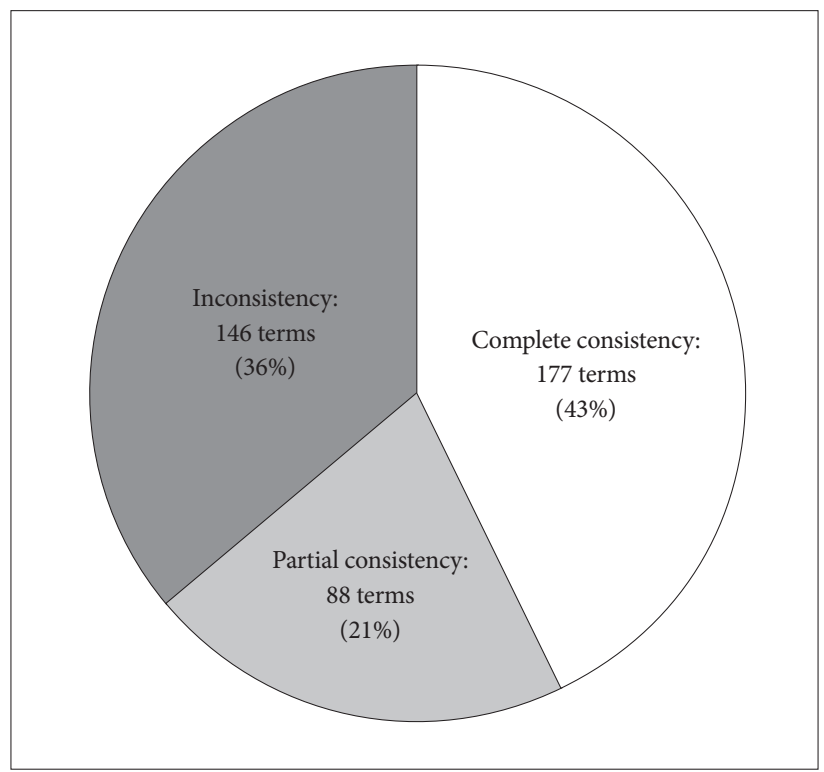

Figure 2. A summary of consistency analysis for the standard term definitions.

dards internationally developed are expected to reduce barriers to cross-cultural communication (Lubinski \& Hudson, 2012) and support reliable multilingualism worldwide (EuroTermBank Consortium, 2006). Successful terminology standards also depend on "high quality in general terms," "harmonization," "exchangeability," "availability," and "speed and up-to-dateness," and sharing a common standardized vocabulary can serve as a starting point for best practices (EuroTermBank Consortium, 2006). Historically, the field of audiology has not employed standard terminology. A previous study (Oh \& Lee, 2016) pointed out issues in audiology terminology and summarized several inconsistent terms, including "hearing loss," "hearing disorder," "central auditory processing disorders," "nonorganic hearing loss," and "sensorineural hearing loss."

The main purpose of the present study was to investigate consistency in audiology-related standard terms by examining the definitions of repeated terms. The results showed 57\% partial or complete inconsistency in term definitions and this implies a current need for improvements in the consistency of standard terms used. Establishing terminology that is consistent for the same terms across various standards should be discussed to improve terminology consistency and establish better audiology-related term data

Above all, the use of consistent terms and definitions may reduce multilingual translation problems in audiology. For instance, Kim \& Lee (2009) addressed several inconsistent audiology terms in South Korea: "auditory steady state response," "auditory brainstem response," "universal newborn hearing screening," "tympanometry," and "audiometer," among others. For successful terminology translations, other reports have suggested terminology translation management systems and provided efficient approaches to improve consistency in multilingual translations (Across Systems GmbH, 2015). In this context, standardized terminology enhances translation quality and efficacy and contributes to reduce translation time and costs for recurring tasks. In addition, research and education based on standardized terms can also enhance their outcomes. This can potentially lead to improving technical documentation, communication in audiology markets, and product and service management. For this reason, development of an international standard in audiology terminology would have a positive impact on those outcomes and the repeated term data investigated in this study can be used as preliminary information for developing standard terminology for audiology.

\section{Ethical Statement \\ N/A}

\section{Acknowledgments}

I would like to express my special thanks to Timothy Kim for his assistance in collecting the data.

\section{Declaration of Conflicting Interests}

There are no conflict interests.

\section{Funding}

This work was supported by a grant from the Korean Ministry of Trade, Industry \& Energy, Project No. 10053685.

\section{REFERENCES}

Across Systems GmbH. (2015). Terminology Workflows: Moving toward Improved Consistency. Retrieved from http://www.across.net/filead$\mathrm{min} /$ sector/knowledge/whitepapers/across-whitepaper-terminologyworkflow-en.pdf.

American National Standards Institute. (2013). American National Standard: Acoustical Terminology (ANSI/ASA S1.1). New York, NY: Acoustical Society of America.

American National Standards Institute. (2015). American National Standard: Bioacoustical Terminology (ANSI/ASA S3.2). New York, NY: Acoustical Society of America.

EuroTermBank Consortium. (2006). Towards Consolidation of European Terminology Resources: Experience and Recommendations from EuroTermBank Project. Riga: Tilde.

IEC 60050-801. (2001). International Electrotechnical Vocabulary-Chapter 801: Acoustics and Electroacoustics. Geneva: International Electrotechnical Committee.

Kim, H. J. \& Lee, H. J. (2009). Proposal of audiologic terminology standardization in Korea. Korean Journal of Audiology, 13(2), 116-127.

Lubinski, R. \& Hudson, M. W. (2012). Professional Issues in Speech-Language Pathology and Audiology. (4th ed.). (pp. 138-156). Boston, MA: Cengage Learning.

Mullen, R. (2010). Clarifying our terminology: Moving the discipline forward by defining terms and sharing data. The ASHA Leader, 15(12), 16-19.

Oh, S. H. \& Lee, J. (2016). A systematic review of audiology terminology. 
Journal of Audiology and Otology, 20(2), 109-113.

Schindler, A. (2005). Terminology in speech pathology: Old problem, new solutions. Advances in Speech Language Pathology, 7(2), 84-86.

Walsh, R. (2005). Meaning and purpose: A conceptual model for speech pathology terminology. Advances in Speech Language Pathology, 7(2), 65 76.

Walsh, R. (2006). Terminology-much more than a definition. ACQuiring
Knowledge in Speech, Language and Hearing, 8(1), 39-41.

Walsh, R. (2008). A framework for effective speech pathology terms. ACQuiring Knowledge in Speech, Language and Hearing, 10(3), 79-83.

Yin, B., Yue, X., Cai, D., \& Zhang, G. (2013). Proceedings from 2013 International Conference on Asian Language Processing: Analysis and Evaluation of Terminology Translation Consistency in Scientific and Technical Literature (pp. 83-86). Urmqi: IEEE. 